Down Syndrome Research and Practice Vol 5, No.1, pp 34 - 38

(C) The Down Syndrome Educational Trust

Printed in Great Britain. All rights reserved.

ISSN: 0968-7912 (1998) 5:1

\title{
TEENAGERS WITH DOWN SYNDROME STUDY ALGEBRA IN HIGH SCHOOL
}

\author{
Elisabetta Monari Martinez
}

\begin{abstract}
This paper deals with the adaptation of an algebra curriculum for two students with Down syndrome who were included in High School. Since the kindergarten, this boy and girl have been fully included in general education classes. This paper examines the rationale for this choice on an algebra program. The adaptation of this program was easy because all that was required was to shorten it and do some additional steps in teaching (a little bit more than in a remedial course). Also, visual prompts were provided to the students. The boy needed a calculator all the time. Both of the students learned to calculate algebraic expressions with parenthesis, with positive and negative numbers and even with powers. The boy was able to do algebraic sum of monomials. The girl performed expressions with fractions. They took written and oral tests at the same time as their classmates, but with different exercises or questions. The girl was able to do some mental arithmetic. Often she was more consistent and careful than her typical classmates. The boy had problems with the integration and he did not attend the school full time. The inclusion, even when it was not perfect, provided the motivation to teach and to learn. In both cases, the crucial point was the daily collaboration of the mathematics teacher with the special educator. Both of the students enjoyed the mathematics program, as many typical students do. Mathematics gave them the fulfilling emotion of succeeding!
\end{abstract}

Keywords: Down syndrome, adolescents, mathematics, alegebra, number, inclusion

Why would an algebra program be useful for a student with Down syndrome?

- Because the mathematics curriculum in these schools begins with a section devoted to algebra and the students with a disability like to participate. As every student, they like to do what their peers do, like to understand what their classmates understand and speak about. This increases the student's self-esteem, as well as, the classmates' regard for him/her. Now they can interact in a field which everybody knows. Unfortunately, adolescents and adults with Down syndrome have a higher probability of having depressive episodes than typical people (Rowitz \& Jurkowski, 1995) and than people with learning disabilities of other aetiology (Collacott et al.,
1992), hence increasing the student's self-esteem can be one of the more important goals.

- Because when a student calculates an algebraic expression, the more frequent logical process needed is to follow a sequence of steps, as a list of instructions, which can also be written in the beginning and kept close by. The persons with Down syndrome usually have no difficulties keeping in mind sequences: they may take time in learning it, but, after they have learned, they can repeat it in the correct order (Kay- Raining Bird \& Chapman, 1994). Since it is then a predicatable process, they enjoy doing it, because it does not cause anxiety, on the contrary, it gives confidence. Obviously, the complexity of the task has to fit the ability of the student. 
- Because the formal language, used to write an algebraic expression, is absolutely well defined and clear. Even if it is concise, it gives each time all the information needed. As soon as the students have understood this formal language, they have no difficulties in following its instructions. An example of an expression, which is not clear, is the one used in elementary schools to write the algorithm of the sum of numbers with many digits: many steps of the algorithm are not written, but kept in mind or written in a way which is not well defined. This algorithm is difficult to understand for typical students and becomes a tough problem for a student with a short working memory span, as it happens in Down syndrome (Hulme \& Mackenzie, 1992; Marcell \& Weeks, 1988).

- Because to get a solution either exactly true or exactly false, but not a matter of opinion, gives self confidence to everybody who performs the task correctly most of the time. This is particularly true for a student with Down syndrome, who often during his/her life performed tasks almost 'well', but seldom 'well' as the peers (for instance with fine motor tasks).

- Because to learn algebra is important for every student, as it is an effective tool to exercise logical skills and is one of the first steps to enter into the mathematical culture.

\section{The Experience.}

D. was a 15 year old girl with Down syndrome, who attended her first grade of Istituto Magistrale (this is a four year school, between the High School and College level, where the students are trained to become elementary school teachers) in Padova, Italy. During all her time in school, she remained in the same classroom with her typical schoolmates. She was supported just 18 hours per week by a special educator, who was a teacher's aid. (In Italy for twenty years, children with any kind of disability have been included, by law, in general education classes and now there are no public special education classes (Ferri, 1987)). D. attended all the regular courses, but the following curricula were adapted: Mathematics, Italian, Latin, English, History, Geography and Sciences. The adaptations were performed by her special educator, together with the teachers of the courses. In all the courses she took the written tests at the same time that her classmates did, but with some different exercises or questions and the tests were graded by the teachers of the courses. In Italian, the special educator often had to guide the development of the written composition, but in mathematics she performed all the exercises without any help and without a calculator. The oral tests were given by the teachers of the courses in front of all the class, as usually. The author, at request of the special educator, prepared D's syllabus for the course of Mathematics, adapting the regular syllabus and was a consultant of the special educator. Just some advice was given to the special educator. She was truly surprised that the program was so easy to implement. The teacher of math had no previous experience with 'special' students, but following the syllabus he helped the special educator (who was not fond of math) to develop it and he prepared all the written tests for D. I think that one of the keys of the success of this program was exactly the continuous cooperation of the special educator with the math teacher. Unfortunately, the teachers of the courses are not always so available for special students!

R. was a 15 year old boy who attended the first grade of an 'Istituto Professionale per geometri' (this is a five year school, between the High School and College level, where the students are trained to become surveyors). He studied Italian, English, History, Sciences, Physics, Mathematics, Chemistry and Technical Design, in adapted programs. He did not attend the school full time and sometimes he did not remain in his classroom. In the school he was assisted by a special educator. (The next year the decision to attend part-time was examined again and was changed. He was fully integrated in the school again!) He learned a lot and, in particular, he had surprising results in mathematics and in design. Also in this case, he took the written tests, at the same time as all classmates did, just with different exercises or questions. In this case also the continuous cooperation of the special educator with the teachers of the courses was crucial for the success of the program.

\section{The mathematics program and the method}

Looking at what D. had learned in the Middle

School and at the regular syllabus of the mathematics course, we prepared the following algebra program for her:

1. Expressions involving the four operations on natural numbers, till three digits.

2. Expressions as in 1 and with one level of parenthesis.

3. Powers of integers.

4. Multiplications and divisions of powers with he same basis. For instance:

$3^{2} \times 3^{3}=3^{2+3}=3^{5}=3 \times 3 \times 3 \times 3 \times 3=243$ $5^{4}: 5^{2}=5^{4-2}=5^{2}=5 \times 5=25$

5. Multiplications and divisions of powers of the same exponent. For instance: $15^{2}: 3^{2}=(15: 3)^{2}=5^{2}=5 \times 5=25$

6. Expressions as in 2, but with two levels of parenthesis. 
7. Expressions as in 6 and with some powers.

8. Expressions as in 6, but with fractions. The fractions involved in the operations of sum and of subtraction had the same denominator. The fractions were simplified. (She had studied fractions in the Middle School).

9. Sum and subtraction of two fractions with different denominators; the new denominator was the product of the two numbers: for instance

$$
\begin{aligned}
& 7 / 2+3 / 4=(7 \times 4) /(2 \times 4)+(3 \times 2) /(4 \times 2)= \\
& 28 / 8+6 / 8=(28+6) / 8=34 / 8=17 / 4
\end{aligned}
$$

10. Positive and negative integer numbers represented on an orientated line. Sum and subtraction.

11. Multiplication and division involving also negative numbers.

12. Expressions as in 6, involving also negative numbers.

13. Expression as in 12, involving also powers.

The program for $\mathrm{R}$. was similar, but with some changes: we dropped the expressions with fractions, also if fractions were involved in the solution of some problems, and we begin the operations with monomials.

In the beginning, for each point, they could have a written list for reference of what they had to do.

(For instance, at point 2, the list could be:

1. Do multiplications and divisions inside the parenthesis;

2. Do the sums and the subtractions inside the parenthesis;

3. Take off the parenthesis;

4. Do the multiplications and the divisions;

5. Do the sums and the subtractions). They could use the calculator. When it was possible, the rules had to be taught with the explanation of the rationale of them: exactly as we do with typical students.

\section{Results}

They learned to calculate algebraic expressions, step by step, following the same path as their typical classmates, but at a slower rate, with some more steps and with individual teaching. About D.: first she learned expressions with integers, then with fractions of the same denominator and later to sum fractions with different denominators, after having reduced them to the same denominator. She learned also to use powers and their properties and later on operations with positive and negative numbers. Regarding the above program, points 12 and 13 were missed for lack of time, but they were done successfully the following year. She performed all the written tests at the same time with the other students and without a calculator, as she was able to do some mental arithmetic. A program of geometry was performed too. The math teacher and the special

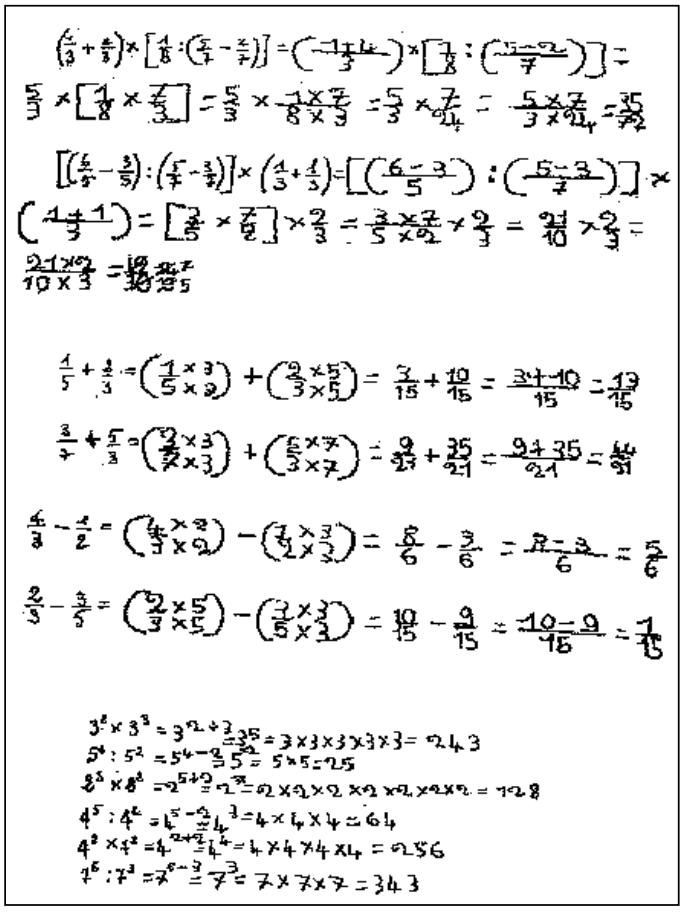

Exercise written by $D$.

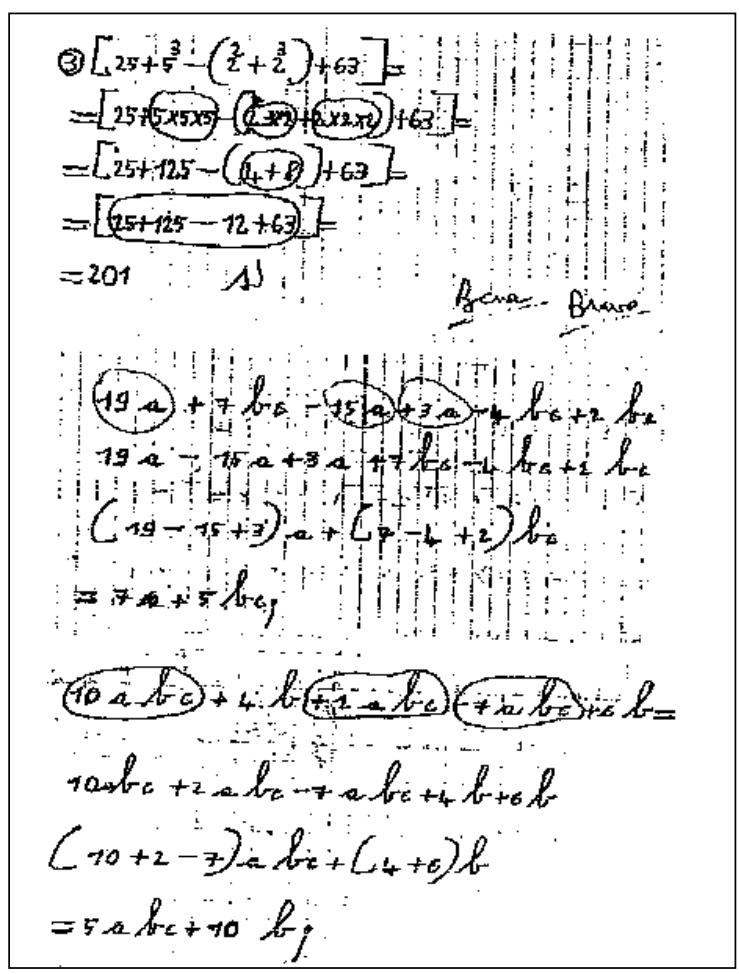

\section{Exercise written by $\mathbf{R}$.}

educator worked together choosing the didactic path and the tests. D. was a willing, adaptable and consistent student: she was graded very well, with 
the maximum grade in mathematics. During the performance of the algebraic expressions, she displayed an autonomous and a critical attitude. In fact, sometimes she decided to cut off some parts of the expression, to solve them aside and to paste the results in the initial expression, in the right place. She did so, in spite of the prohibition of the teacher, who considered it a non-advantageous operation, even if clever, as it often led to misprints in the transcription. The teachers observed her improved personality, lexicon and more general communication skills in that year. These results are testified by the documents of the school.

About R.: first he learned to perform expressions with integers and then with positive and negative integer numbers, with two levels of parenthesis and powers, algebraic sum of monomials and problems of geometry on the plane. He was not able to do any mental arithmetic (even if he was able to do it in previous years) and he used a calculator all the time. He took all the written tests with the other students. At school he displayed some behavioural problems, maybe due to the partial integration in the school: this was the first time in his life he was not fully integrated in the school and he suffered from this exclusion. The next year, he asked for and obtained full inclusion: no behavioural problem occurred. $\mathrm{He}$ was a willing and consistent student, but surely not all his potentialities were developed. He liked mathematics all the time and enjoyed doing it.

\section{Discussion}

What are the reasons for these results?

- To have no prejudice about what a person with a disability can learn, but to believe that, if we are patient and find the right way of teaching, there are no limits to what people with disabilities can learn. These teenagers are just typical Down syndrome students (for instance the mental age of the girl is 6.5 years and her IQ is 45 in the WISC-R test) with very typical Italian families. They always trusted in their childrens' possibilities in a realistic and consistent way and they always were watching out, looking for the best.

- The strength and the consistency of these students, who, as many persons with Down syndrome, can work a lot, if they are motivated, despite of their difficulties.

- The inclusion in a regular class, which gives to the students the motivation, to adapt themselves to their surroundings and to improve both their social and their academic skills. At the same time, the inclusion stimulates the teachers to try more 'normal' programs with special students.....and often it works?
- The professional ability of the special educator, who provided a mediated learning experience (Kozulin \& Falik, 1995; Kozulin \& Presseisen, 1995) to the student, as a tutor, but inside the classroom (Ferri, 1987). To succeed in it, the special educator needs good training as a teacher.

- The collaboration between the teacher of the course and the special educator in adapting the mathematics curriculum and in teaching it. They need to have both good training and the philosophy that the special students are not just a matter of the special educator, but they are students of the class and then they need the attention and the work of all the teachers.

- The choice of the mathematics curriculum and of its progression. In this choice we have to consider the syllabus of the course, the previous curriculum of the student, his/her interests, strengths and weaknesses, so that in the progression we have to alternate topics of high interest and easier to understand with more difficult topics. Sometimes, what was hard for us, is not so hard for him/her and vice versa. This means that we have to know the pathology of the student (as well as we can), we have to master the topic with the aim of preparing a right adaption (which is not always a reduction!), and we need to be flexible to modify the program, if it does not work.

\section{Conclusions}

A student with mental retardation can succeed in academic programs, where even typical students may have difficulties, and can enjoy studying these programs. If we believe the academic culture is precious and pleasing for us, why should we not share it with people with difficulties? If it helps us, why should it not help them? I think the right path might be a fair balance between academic programs and trainings for the autonomy.

\section{Author:}

ELISABETTA MONARI MARTINEZ, Dr., Researcher, Dipartimento di Matematica Pura ed Applicata, Universita degli Studi di Padova, via Belzoni, 7, 35131 Padova, Italy.

E-mail: martinez@math.unipd.it 


\section{References}

Collacott, R.A., Cooper, S.A. \& McGrother, C. (1992). Differential rates of psychiatric disorders in adults with Down's syndrome compared to other mentally handicapped adults. British Journal of Psychiatry, 161, 671-674.

Ferri, R. (Ed.).(1987) Il bambino Down a scuola. Seminario di studi. Roma, ottobrg 1985. Bergamo (Italy): Juvenilia.

Hulme, C. \& Mackenzie, S. (1992). Working memory and severe learning difficulties. Hove (U.K.): Lawrence Erlbaum Associates Publishers.

Kay-Raining Bird, E. \& Chapman, R. (1994) Sequential Recall in Individuals With Down Syndrome. Journal of Speech and Hearing Research, 37, 13691380.

Kozulin, A. \& Falik, L. (1995) Dynamic cognitive assessment of the child. Current Directions in Psychological Science, 4 (6), 192-196.

Kozulin, A. \& Presseisen, B.Z. (1995) Mediated learning experience and psychological tools: Vygotsky's and Feuerstein's perspectives in a study of student learning. Educational Psychologist 30 (2), 67-75.

Marcell, M.M. \& Weeks, S.L. (1988) Short-term memory difficulties in Down's syndrome. Journal of Mental Deficiency Research, 32, 153-162.

McDonnell, J., Thorson, N., McQuivey, C. \& Kiefer-O'Donnell, R. (1997) Academic engaged time of students with low-incidence disabilities in general education classes. Mental Retardation, 35 (1), Feb., 18-26.

Nadel, L. (Ed.) (1988) The psychobiology of Down Syndrome, Cambridge: MIT Press.

Nadel, L. \& Rosental. D. (Eds.), (1995) Down Syndrome: Living and Learning in the Community, New York: Wiley-Liss, Inc.

Pueschel, S., Gallagher, P.. Zartier, A. \& Pezzullo, J. (1987) Cognitive and learning processes in children with Down syndrome. Research in Developmental Disabilities, 8, 21-37.

Rowitz, L. \& Jurkowski, E. (1995). The myth and realities of depression and Down syndrome. In L.Nadel \& D.Rosental (Eds) Down Syndrome: Living and Learning in the Community (pp.50 57). New York: Wiley-Liss, Inc.

Snell, M.E. (1990). Schools are for all kids: The importance of integration for students with severe disabilities and their peers. In J.W.Lloyd, N.N.Singh \& A.C.Repp (Eds), The regular education initiative: Alternative perspectives on concepts, issues and models (pp.133-149) Sycamore, IL: Sycamore. 